\title{
LA CLASSIFICATION DU CONTENU CONCEPTUEL DES CURRICULUMS CONCERNANT L'ÉNERGIE COMME OUTIL ÉDUCATIF
}

\author{
Dimitris Koliopoulos ${ }^{1}$ \\ Jean Marie Boilevin ${ }^{2}$ \\ Konstantinos Ravanis ${ }^{1}$ \\ (1) Université de Patras, Grèce \\ dkoliop@upatras.gr, ravanis@upatras.gr \\ (2) UMR ADEF ; IUFM Aix-Marseille, France \\ jean-marie.boilevin@aix-mrs.iufm.fr
}

\section{Resumo}

Nos últimos anos as pesquisas sobre as dificuldades de ensino e de aprenidzagem das ciências físicas têm condizido o desenvolvimento a Didáticas das Ciênicas Físicas como um campo cientítifo relativamente autônomo. O quadro de pesquisa e de aplicação proposto por esse campo tem influenciado um certo número de modificações em diversos sistemas educativos. Esse artigo apresenta uma discussão e uma tentativa de modelização de propostas curriculares abordando o conceito de energia. È desenvolvida a hipótese de que a passagem de uma abordagem empírico-descritiva da estrutura, do conteúdo e da aplicação do conceito de energia a um estudo que utiliza conceitos fundamentais oriundos da pesquisa em ensino de Física oferece novas perspectivas de ensino.

Palavras-chave: ensino de física, curriculums, energia.

\author{
ABSTRACT
}

Key-words:

\section{INTRODUCTION}

Au cours de ces dernières décennies, l'étude des difficultés d'enseignement et d'apprentissage des sciences physiques a conduit au développement de la Didactique des sciences physiques, c'est-à-dire à la création d'un champ scientifique relativement autonome, puisqu'il constitue désormais un cadre de recherche et d'application déterminé (Astolfi \& Develay, 1989). Parallèlement au développement de la Didactique des sciences physiques, ont été réalisés un certain nombre de modifications concernant l'enseignement de celles-ci et cela dans les divers systèmes éducatifs. Or très souvent, les changements de programmes, de manuels scolaires, de méthodologies éducatives ne sont influencés ni par les schémas théoriques ni par les résultats des recherches en Didactique des sciences physiques. On pourrait sans doute rechercher des explications quant à l'interprétation de cette réelle absence de communication dans l'écart entre le cadre épistémologique au 
sein duquel se développe la Didactique des sciences physiques, et "l'épistémologie" d'une éducation quotidienne, qui impose les conditions et les contraintes des pratiques éducatives (Develay, 1992).

La formation de la Didactique des sciences physiques peut être décrite comme un parcours sur lequel travaille la communauté scientifique, et cela autour d'une série d'hypothèses de départ relatives aux processus d'apprentissage, aux caractéristiques de l'enseignement, et à la nature de la science. Le cadre dans lequel se développe l'enseignement des sciences physiques est défini par le genre du curriculum, les contraintes matérielles imposées par les conditions réelles de travail, et par les conceptions des enseignants quant à leur travail, les sciences physiques et l'apprentissage. Si on suppose, en effet, que la communauté scientifique réalise sa recherche en ayant comme objectif principal l'amélioration de l'enseignement des sciences physiques, alors la nécessité d'une recherche des possibilités de rétablissement de la communication entre ces deux cadres - une recherche qui peut être réalisée à divers niveaux et au moyen de diverses techniques - devient évidente (Martinand, 1994 ; Louden \& Wallace, 1994).

Cependant cette tentative de développement de la communication entre recherche et pratique pédagogique, présuppose la création d'une formule qui permettra la formation d'un champ de référence commun entre chercheurs et enseignants. Cette formule représente un modèle qui devrait jouer le rôle de médiateur entre les deux cadres et devrait être élaboré de façon à ce qu'il soit lisible et utilisable aussi bien par les enseignants que par les chercheurs. Dans cet article, nous présentons un tel modèle, dans lequel nous opérons une classification des curriculums concernant le concept d'énergie au

niveau du collège ${ }^{1}$. Cette classification constitue une tentative de modélisation d'une grande série de programmes d'enseignement, pour lesquels nous supposons que le passage d'une approche descriptive empirique de la structure, du contenu et du champ d'application du concept d'énergie, à une étude en fonction de l'utilisation de concepts fondamentaux du cadre de la Didactique des sciences physiques, offre certaines possibilités et perspectives à la communication souhaitée (Koliopoulos \& Ravanis, 1999). Nous supposons ainsi qu'un tel modèle pourrait, en vue de l'élucidation des conditions de travail au collège concernant l'enseignement de l'énergie, permettre sans doute aux chercheurs d'apprécier la signification des matériels et des décisions des enseignants, la codification et l'analyse des pratiques pédagogiques et des activités didactiques. De plus, ce modèle devrait aider les enseignants à prendre conscience de la cohérence de leurs choix, de mieux mettre en valeur les matériels et les manuels scolaires, et engendrer la rationalisation des réorganisations possibles de leur travail.

\section{LA CLASSIFICATION DES CURRICULUMS}

La classification à laquelle nous nous référons, n'est autre qu'un regroupement de conclusions provenant de l'analyse du contenu de programmes d'enseignement précis, qui constituent des approches connues et valides d'une série de curriculums de divers pays. Le principal critère de choix de ces programmes est leur caractère d'introduction du concept d'énergie au niveau du collège. Cette classification ne correspond à aucun curriculum précis, mais, comme nous l'avons déjà précisé, il représente une sorte de modèle théorique qui met à disposition un ensemble de caractéristiques générales pouvant se manifester entièrement ou partiellement dans certains programmes d'enseignement. Ces caractéristiques générales proviennent de conceptions explicites ou implicites, qui sont décrites en termes de notions de la recherche réalisée dans le cadre de la Didactique des sciences physiques, alors qu'il est en même temps possible d'expliquer des éléments de la structure, du contenu et des activités proposées par les curriculums. C'est ce second fonctionnement "explicatif" de notre classification qui devrait intéresser principalement les enseignants, mais aussi les didacticiens dont l'objet de recherche est la formation des enseignants.

${ }^{1}$ En Grèce, le collège concerne des élèves de 12 à 15 ans et en France, il accueille des élèves de 11 à 15 ans environ. 
La classification proposée comporte trois catégories de curriculum : le curriculum "traditionnel", le curriculum "innovatif" et le curriculum "constructiviste". La nature et les caractéristiques de ces catégories seront décrites de façon analytique par la suite.

\subsection{Le curriculum "traditionnel"}

Nous allons utiliser en tant que représentants du curriculum "traditionnel" concernant l'énergie, le programme d'enseignement anglais bien connu Physics for you (Johnson, 1994) ainsi que celui de physique en vigueur au collège grec (Zenakos et al., 1994). L'intention explicite ou implicite du curriculum traditionnel est que les élèves comprennent les sujets qui touchent principalement, pour ne pas dire uniquement, au contenu des sciences physiques. La conséquence d'une telle intention est que le curriculum traditionnel envisage tous les concepts de sciences physiques de la même façon. Par exemple dans le "Guide de la matière à enseigner et l'enseignement des cours au collège et lycée", qui accompagne le programme grec, il n'existe pas d'objectifs spécifiques concernant les divers concepts de physique. Aucun principe, aucune loi ou concept n'a de caractère privilégié. De son côté, le programme d'enseignement anglais reconnaît que le concept d'énergie constitue un concept scientifique fondamental - un chapitre introductif sur le sujet ("Énergie") a d'ailleurs été inclus - sans pourtant qu'il acquière une importance fonctionnelle particulière dans la structure et le contenu du reste du programme.

Une des caractéristiques principales du curriculum traditionnel qui découle de l'intention précédente, est la dispersion du concept d'énergie à travers les différentes unités thématiques. Dans le programme grec par exemple, nous rencontrons le concept dans des unités thématiques très différentes comme "Travail", "Machines simples", "TempératureChaleur", "Théorie cinétique des gaz", "Machines thermiques", et "Émission et propagation de la lumière". Il en va de même dans le programme d'enseignement anglais ("Thermomètres", "Travail-Énergie-Puissance", "Machines simples", "Effets thermiques du courant électrique", "Radioactivité", par exemple). La manière fortuite d'introduire et d'analyser divers concepts physiques et, par conséquence, celui d'énergie, semble être due au fait que même les sujets du curriculum traditionnel sont introduits de façon fortuite, puisque il n'existe pas d'autre critère extérieur - objectif didactique - hormis la compréhension du contenu de la science. Une des conséquences majeures de la caractéristique du curriculum traditionnel mentionné précédemment, est que l'étude du concept d'énergie devrait être réalisée dans des cadres conceptuels différents, et que dans chacun d'eux, le concept d'énergie obtienne un sens systémique et empirique différent, c'est-à-dire une autonomie conceptuelle relative (Baltas, 1990). Ainsi, dans les unités thématiques des programmes grec et anglais sont juxtaposés et/ou sont mélangés les cadres conceptuels de la Mécanique, de la Calorimétrie, de la Thermodynamique, de la Mécanique Statistique, de l'Électricité, etc. Il semble qu'au niveau du collège, aucun rapport n'existe entre ces différents cadres conceptuels. Résultat : l'étude du concept d'énergie représente chaque fois une approche indépendante. En s'appuyant sur cette analyse, la conclusion à laquelle on pourrait aboutir est que la juxtaposition de nombreux cadres conceptuels qui touchent au concept d'énergie et/ou le mélange de tels cadres dans de petites unités thématiques, réduit le fonctionnement du concept puisque à chaque fois l'élève est confronté à un sens différent du même concept. Et c'est ainsi qu'augmente le risque de tomber dans des malentendus conceptuels. La juxtaposition et/ou le mélange des différents cadres conceptuels ne concernent pas uniquement le concept d'énergie. Il semblerait qu'il s'agisse d'une caractéristique plus générale du curriculum traditionnel qui découle des intentions et objectifs mentionnés plus haut. Un autre exemple de confusion des différents cadres conceptuels qui se réfère à un autre concept de moindre importance que celui de l'énergie, est celui de la pression. Dans le programme d'enseignement grec par exemple, la pression est introduite comme une tension mais aussi comme fonction de point (Kariotoglou et al., 1990).

Une deuxième caractéristique essentielle du curriculum traditionnel, qui est intimement liée à la précédente, est que le concept d'énergie est introduit soit en tant que concept dérivé du travail (dans le cadre conceptuel de la Mécanique, par exemple) soit en tant que fonction des grandeurs observables qui décrivent le champ mono-phénoménologique d'application du concept (comme par exemple les cadres conceptuels de la Calorimétrie et de l'Électricité). Dans le curriculum grec, l'énergie est d'emblée introduite auprès des phénomènes mécaniques au moyen du concept de travail (dans 
l'unité "Énergie-Formes d'énergie"), alors que plus tard, la chaleur (énergie) absorbée par une quantité d'eau qui se réchauffe est liée à la masse, la chaleur spécifique et la variation de la température du liquide (dans la partie "Calorimétrie"). Nous remarquons une organisation inverse dans le curriculum anglais où l'approche des phénomènes thermiques (dans la partie "Mesure de la chaleur") précède l'approche des phénomènes mécaniques (dans le chapitre "Travail-Énergie-Puissance"). La caractéristique de l'introduction du concept d'énergie, en tant que fonction des grandeurs, s'observe également dans un ancien programme d'enseignement français au niveau collège, où l'unique cadre conceptuel est celui de l'électricité (Gaborieau et al., 1994., Durandeau et al., 1994). La principale critique adressée au contenu conceptuel du curriculum traditionnel, concerne son noyau dur conceptuel principal qui n'est autre que la définition de l'énergie en tant que concept dérivé dans le cadre de la Mécanique. Cette approche est considérée comme insuffisante et fausse selon des critères scientifiques, sociaux et psychologiques (Lehrman, 1973; Arons, 1990). De même, les résultats des recherches empiriques qui se réfèrent aux conceptions des élèves quant au concept d'énergie, confirment ce que les enseignants des sciences physiques connaissent très bien, c'est-à-dire que l'approche énergétique des phénomènes mécaniques est difficilement compréhensible par la majorité des élèves (Solomon, 1992 ; Driver et al., 1994). De plus, l'introduction du concept d'énergie en tant que fonction de mesures physiques observables limite l'approche énergétique au niveau quantitatif, alors que nous savons qu'une sorte de rapprochement entre les conceptions qualitatives pré-énergétiques des élèves et la nature quantitative de l'énergie, est nécessaire (Lemeignan \& Weil-Barais, 1993) pour une meilleure compréhension.

Une troisième caractéristique du curriculum traditionnel est la sélection fortuite du champ d'application des concepts physiques, c'est-à-dire du champ des phénomènes qui fonctionnent en tant qu'applications du cadre conceptuel en question. Ceci semble être une conséquence supplémentaire de la dispersion des concepts physiques dans les diverses unités thématiques $\mathrm{du}$ curriculum. Tous les phénomènes physiques constituent pour le concept d'énergie le champ phénoménologique d'application potentielle. Tout phénomène est au service de l'étude du champ conceptuel concerné avec lequel le concept d'énergie est en corrélation. Ceci provoque parfois une étude conceptuelle différente de certains phénomènes physiques mêmes s'ils sont thématiquement proches. Par exemple, le pendule simple, aussi bien dans le curriculum grec que dans le curriculum anglais, est utilisé comme un phénomène physique adéquat à l'introduction du principe de conservation de l'énergie mécanique sous la forme "l'addition de l'énergie cinétique et de l'énergie potentielle reste invariable". Mais dans les unités suivantes, l'étude des machines simples se fait sous la forme du principe de conservation de l'énergie, où la machine simple est considérée comme un système ouvert d'entrée-sortie de l'énergie. Ces exemples montrent précisément la relation fragile du cadre conceptuel et du champ d'application quant au concept d'énergie, qui est le résultat d'un choix fortuit de sujets et de concepts, comme nous l'avons développé dans les paragraphes précédents.

\subsection{Le curriculum "innovatif"}

Depuis la fin des années 60, des programmes d'enseignement qui s'appuient sur des restructurations conceptuelles du contenu des sciences physiques sont apparus. Celles-ci favorisent dans ces programmes la revalorisation du concept d'énergie. Le curriculum innovatif se fonde sur ces restructurations qui reconnaissent d'une part l'importance fondamentale du principe de conservation de l'énergie en sciences physiques, et, d'autre part, le caractère unificateur et transphénoménologique du concept d'énergie. En tant que représentants de cette tendance du programme innovatif, nous présentons le programme américain "Energy" qui a été développé par Haber-Schaim (1983), ainsi qu'une de ses formes modifiée et développée en Israël par Shadmi et ses collaborateurs (Shadmi et al., 1978). Une deuxième tendance du programme innovatif apparaît à la fin des années 70, quand la crise du pétrole mobilise les systèmes éducatifs des pays industriellement développés, qui réagissent en élaborant des curriculums dans lesquels se trouve accentuée non seulement l'importance primordiale du concept d'énergie pour les sciences physiques, mais aussi son importance sociale, puisqu'elle est intimement liée à des problèmes comme celui de l'économie d'énergie. En tant que représentants de cette tendance du programme innovatif, nous présentons le programme français "Sciences Physiques, Libres Parcours" (Agabra et al., 1979), et le programme allemand "Neue Physik Das Energiebuch" (Falk \& Herman, 1981). 
Dans le curriculum innovatif, nous passons donc de la dispersion du concept d'énergie en diverses unités thématiques, à une plus large unité conceptuelle ou même au fait de considérer l'énergie comme principe organisateur du curriculum en entier. Ainsi, le principal élément unificateur de la série d'unités qui apparaît dans le programme "Energy" (par exemple, dans les unités successives "Chaleur", "Chaleur et charge électrique", "Où se trouve la chaleur ?" et "Énergie potentielle"), est le concept de transformation des formes d'énergie de façon à ce que soit confirmé le principe fondamental de la conservation de l'énergie. Le programme israélien est, lui aussi, basé sur les deux principes suivants : " a. Au cours de toute interaction entre deux phénomènes, “quelque chose" (que nous nommons énergie) est transféré de l'un à l'autre, et, b. à chaque transfert d'énergie ou à chacune de ses transformations, la quantité totale d'énergie demeure stable". De même, “chaque nouvelle forme d'énergie est définie quantitativement à travers l'étude de sa conversion en d'autres formes d'énergie" (Shadmi et al., 1978). Une semblable organisation conceptuelle du contenu est aussi observée dans les programmes français et allemand où le concept de transfert d'énergie joue surtout le rôle unificateur principal dans l'étude des diverses activités. Et ceci est particulièrement renforcé dans le programme français par l'étude du concept de puissance (dans les parties "Débit d'énergie" et "Pertes et rendement", par exemple) et dans le programme allemand avec l'introduction du concept du porteur d'énergie qui “s'écoule”, en même temps que la quantité d'énergie transférée des sources d'énergie vers les récepteurs d'énergie (par exemple dans les unités successives "Sources d'énergie et récepteurs d'énergie ", "Porteurs d'énergie", "Le porteur d'énergie : électricité", “Le porteur d'énergie : moment d'inertie", etc.).

Les intentions particulières et les objectifs du curriculum innovatif ont un retentissement non seulement sur la structure du programme, mais aussi sur son contenu conceptuel. Dans le cas de la tendance du curriculum innovatif où le rôle principal est joué par le contenu de la science, l'énergie est introduite en tant que concept primordial alors qu'est souligné son caractère unificateur et trans-phénoménologique, qui est assuré à travers le principe de conservation de l'énergie. L'étude de la chaleur par exemple, en tant que fonction de la masse, de la chaleur spécifique et de la variation de température d'une quantité de liquide réchauffé, qui, dans le curriculum traditionnel représentait un objet d'étude autonome, acquiert désormais du sens, uniquement à travers le processus de transformation d'une autre forme d'énergie en chaleur (dans le cas, par exemple, du réchauffement d'une quantité d'eau à l'aide du mouvement d'une aile provoqué par la chute d'un corps (Shadmi et al., 1978). Dans le cas de la deuxième tendance du curriculum innovatif, où les intentions scientifiques et sociales suggèrent un ensemble plus équilibré d'objectifs didactiques, l'énergie est également présentée comme un concept primordial alors qu'en même temps est choisi le cadre théorique de la Thermodynamique en tant qu'unique cadre conceptuel de référence. Dans les programmes d'enseignement en question, la forme que prend la transformation didactique du cadre conceptuel de la thermodynamique est celle de divers modèles de la chaîne énergétique. Dans le programme français, selon ce modèle, l'énergie est introduite en tant que mesure physique commune qui caractérise des types différents de réservoirs d'énergie, même si elle est présentée sous différentes formes. Parallèlement, une distinction claire est faite entre l'énergie qui décrit la situation d'un système et le transfert d'énergie qui dénote la phénoménologie de transfert d'énergie. Quatre formes de transfert d'énergie sont définies : le travail mécanique, le travail électrique, la chaleur, et le rayonnement. De même, le principe de conservation de l'énergie est suivi du concept de dégradation d'énergie, imposé plutôt par l'approche technologique et les exigences sociales (voir par exemple la partie "Machines thermiques") plutôt que par l'approche scientifique du concept. Les précédentes caractéristiques conceptuelles proposent un modèle "énergétique" explicatif qui décrit et interprète un grand nombre de phénomènes physiques ayant des caractéristiques mécaniques, électriques, magnétiques et chimiques. En ce qui concerne le programme allemand, l'étude de l'énergie se fait à travers le cadre d'un modèle de chaîne énergétique, où le flux d'énergie se manifeste entre des sources d'énergie, des récepteurs et des transformateurs d'énergie, et s'accompagne du flux d'au moins une grandeur extensive (porteur d'énergie) qui indique le mécanisme de transfert de l'énergie. Le modèle conceptuel précédent s'applique tout comme dans le programme français à un grand nombre de phénomènes physiques, où les systèmes physiques proviennent surtout d'un environnement physique et technologique familier aux élèves.

Dans le curriculum innovatif le champ d'application du concept d'énergie est étendu, comme cela arrive d'ailleurs dans le programme traditionnel, à cause de la nature trans-phénoménologique du concept. Cependant, dans le cas du 
programme innovatif, ce champ sert surtout le rôle unificateur du concept d'énergie. Ainsi, les mêmes phénomènes physiques, dont l'étude dans le programme traditionnel exige des cadres conceptuels différents où l'énergie acquiert des significations différentes, sont, dans le programme innovatif, abordés conceptuellement de la même façon. Dans le programme israélien par exemple, le champ d'application est trans-phénoménologique et sert toujours l'étude des transformations et de la conservation de l'énergie. Dans ce programme, est tentée "pas à pas" une approche expérimentale qualitative et quantitative des diverses formes d'énergie à travers l'étude de leur transformation. Ainsi, nous rencontrons des phénomènes physiques comme ceux de la remontée d'un corps à l'aide d'une roue de vélo, la remontée d'un corps à l'aide d'un appareil adéquat dans lequel est incorporé un thermomètre sensible pour mesurer l'augmentation de la température, le réchauffement de l'eau avec un thermoplongeur etc., phénomènes qui nous aident à déduire les relations qui relient les différentes formes d'énergie avec les grandeurs physiques relatives et observables mais aussi les différentes formes entre elles. Le champ d'application du programme français est principalement trans-phénoménologique. Une des idées principales est celle de demander aux élèves de reconnaître l'énergie comme élément commun aux différents phénomènes physiques rencontrés, où la cause et le résultat sont identiques. Par exemple, on leur demande de définir la particularité commune dans les différents cas d'allumage d'une ampoule (à l'aide d'une pile par exemple, ou d'une dynamo qui fonctionne grâce à la chute d'un corps, ou encore à l'aide d'un thermocouple, etc.). Une autre suite de phénomènes utilisant une pile peut jouer exactement le même rôle. Les caractéristiques trans-phénoménologiques du champ d'application dans la deuxième intention du curriculum innovatif sont explicables non seulement parce qu'elles servent le cadre conceptuel des chaînes énergétiques (qui par nature contient le transfert et le stockage de l'énergie en réservoirs ayant des caractéristiques transphénoménologiques différentes) mais aussi parce que le champ d'application se réfère à des sujets et des problèmes de la vie quotidienne et de la technologie qui généralement ont un caractère trans-phénoménologique. Dans le guide pédagogique du programme allemand, est mentionné en effet que la technologie ne joue pas le rôle d'application des lois physiques mais, qu'au contraire, de nombreux problèmes technologiques soutiennent l'apprentissage des concepts physiques.

\subsection{Le curriculum "constructiviste"}

Il semble évident que l'approche constructiviste de l'apprentissage et de l'enseignement ne forme pas encore de modèle global d'enseignement et d'élaboration d'un curriculum. En effet, les recherches actuelles autour de l'élaboration de programmes d'enseignement constructivistes servent divers objectifs de recherche et s'appuient sur différentes conceptions relatives à l'incorporation dans l'enseignement et le curriculum de conclusions scientifiques se référant aux conceptions des élèves à propos des concepts physiques. Le curriculum constructiviste auquel nous nous référons, concerne des approches qui semblent former des propositions complètes d'enseignement de l'énergie et non pas des approches restreintes, où le processus d'élaboration du concept en fonction d'un nombre limité d'activités est examiné. Les intentions communes du curriculum constructiviste sont : a) la nécessité d'une intégration organique des conceptions des élèves pour les concepts physiques dans la formulation d'intentions et d'objectifs didactiques et, b) la formulation d'objectifs didactiques de façon à laisser transparaître les processus d'élaboration conceptuelle, ce qui n'est pas le cas dans les objectifs clairement exprimés du curriculum traditionnel et innovatif.

Une analyse systématique des diverses recherches relatives aux programmes constructivistes d'enseignement (Koliopoulos, 1997), a montré qu'il existe au moins deux approches dont les caractéristiques peuvent être généralisées de sorte que nous puissions parler de curriculum constructiviste. La première approche se réfère à des programmes ayant pour but de permettre aux élèves de différencier certains concepts proches relatifs au concept d'énergie qui, avant l'enseignement, étaient utilisés de façon indifférenciée. Dans ce cas, des activités didactiques favorisant la disparition d'obstacles conceptuels chez les élèves sont introduites, et conduisent à la différenciation progressive des concepts en question. Cette approche peut être également mono-phénoménologique. Des représentants caractéristiques de cette tendance du curriculum constructiviste sont le programme d'enseignement de Agabra (1986) qui a pour but la différenciation entre les notions de température, chaleur et énergie, et le programme de Shipstone \& Gunstone (1985) où est tentée la différenciation des notions d'énergie 
électrique et de courant électrique. La deuxième approche se réfère à des programmes d'enseignement qui s'appuient sur l'interaction des premières conceptions des élèves, et "d'un modèle - germe" explicatif ayant pour but l'élaboration d'une forme plus évoluée de ce modèle. Cette approche exige l'activation du syllogisme causal et linéaire "source - action récepteur", que les élèves utilisent très fréquemment quand ils tentent de décrire et/ou d'expliquer le fonctionnement de divers systèmes physiques (Tiberghien, 1989). Il s'agit d'une approche trans-phénoménologique. En tant que représentants de cette tendance du curriculum constructiviste, nous nous référons aux programmes d'enseignement de Lemeignan \& Weil-Barais (1990), Tiberghien \& Megalakaki (1995) et Koliopoulos \& Ravanis (2000a) dans lesquels est tentée l'élaboration des particularités des divers modèles de la chaîne énergétique.

Les objectifs didactiques dans les deux tendances du curriculum constructiviste influencent plus ou moins, aussi bien l'organisation et la nature du contenu conceptuel du programme, que son champ d'application phénoménologique. Le programme d'Agabra propose ainsi un plan d'élaboration progressive des notions de température, chaleur et énergie, depuis l'école primaire jusqu'au lycée. L'introduction de la chaleur en tant que forme de transfert d'énergie est proposée à la fin du premier cycle d'études de l'enseignement secondaire (collège) et/ou au début du deuxième cycle (lycée). Elle a pour but de dépasser l'obstacle gnostique selon lequel la chaleur est considérée comme une sorte de substance, et plus particulièrement de dépasser la difficulté d'élaboration de notions équivalentes comme chaleur et travail, observée chez des élèves de cet âge (Agabra, 1986). Dans le programme de Shipstone \& Gunstone, la notion d'énergie électrique est d'abord introduite car elle apparaît plus proche des conceptions des élèves par opposition aux programmes traditionnels (Shipstone \& Gunstone, 1985). Les programmes de la deuxième tendance s'appuient sur l'activation du syllogisme causal linéaire "source-action-récepteur" qui a pour résultat la formulation de conceptions que certains nomment conceptions pré-énergétiques, compatibles avant tout avec divers modèles de la chaîne énergétique. Le changement de ces conceptions qualitatives en quantitatives est un des objectifs didactiques principaux de ces programmes. Ainsi dans le programme de Koliopoulos \& Ravanis (2000a), comme il a été constaté que les élèves expriment plus facilement des conceptions pré-énergétiques dans les phénomènes thermiques plutôt que dans les phénomènes mécaniques, l'introduction du "modèle - germe" se fait d'abord à travers les phénomènes thermiques. Son élaboration auprès des phénomènes mécaniques est ensuite tentée au moyen de ses propriétés analogiques.

Un élément essentiel de la nature des cadres conceptuels dans les deux tendances du curriculum constructiviste est qu'il insiste tout d'abord sur les éléments qualitatifs de divers concepts en renforçant ainsi les conceptions préénergétiques des élèves qui progressivement évoluent vers des cadres quantitatifs. Par exemple, dans le programme de Shipstone \& Gunstone (1985), est d'abord favorisée la conception pré-énergétique des élèves qui décrivent le fonctionnement d'un circuit électrique simple, comment la pile donne "l'énergie" que la lampe consomme. Alors seulement est introduite la notion de courant électrique contenant des éléments qualitatifs (comme, par exemple, la direction qui provient de la description des effets magnétiques) et quantitatifs (comme les indications des ampèremètres). Dans le programme de Lemeignan \& Weil-Barais (1990) différents systèmes physiques sont introduits comme le circuit simple lampe-ampoule, l'imitation d'une voiture électrique, la lampe qui s'allume à l'aide d'une dynamo et de la chute d'un corps, etc. Les élèves doivent les décrire et expliquer leur fonctionnement, tout d'abord au moyen de termes qualitatifs de la chaîne énergétique. Par la suite, ils sont invités à réfléchir sur la modification à apporter à un montage pour faire varier un effet produit (que faire par exemple, pour que la lampe s'allume de la même manière plus longtemps ? Ou bien, que faire pour que la voiture aille plus loin ?). L'utilisation du concept d'énergie en tant que quantité est alors rendu obligatoire. L'élaboration d'éléments de divers modèles de la chaîne énergétique représente, de toute façon, une des caractéristiques principales de la deuxième tendance du curriculum constructiviste. Le fonctionnement de ce cadre conceptuel semble être dû, tout d'abord, à la compatibilité du modèle avec le champ initial des conceptions des élèves pour le concept d'énergie, à savoir les conceptions pré-énergétiques. Cette compatibilité est due tant à des ressemblances syntaxiques qu'au genre de l'explication donnée : explication causale selon laquelle la cause est en rapport avec les facteurs extérieurs du changement du système physique étudié (Halbwachs, 1973). Elle est également due à sa puissance heuristique. Cette dernière peut être résumée : a) à sa capacité de fonctionnement précis tant au niveau qualitatif qu'au niveau quantitatif, fait qui contribue considérablement au processus de 
transformation des concepts pré-énergétiques qualitatifs en concepts énergétiques quantitatifs, et, b) à ses propriétés analogiques qui permettent l'étude énergétique de divers champs de phénomènes à travers le même cadre conceptuel.

Les objectifs didactiques du curriculum constructiviste semblent aussi tout particulièrement influencer le champ phénoménologique d'application du concept d'énergie. Ceci est dû aux activités prévues qui ont désormais pour but de renforcer un certain nombre de conceptions des élèves, ou de contribuer au dépassement de certains obstacles conceptuels. Ce qui a pour résultat de limiter théoriquement l'inépuisable champ d'application du concept, et ceci à cause des changements conceptuels recherchés. De même, ce champ apparaît non seulement comme un élément indépendant qui jouerait simplement le rôle de support phénoménologique, mais aussi comme un élément intimement lié à ses caractéristiques conceptuelles, afin d'élaborer un cadre conceptuel. C'est ainsi que sont mises en évidence des catégories de phénomènes physiques considérées comme non privilégiées en tant que champs d'application du contenu conceptuel en question. De tels phénomènes sont, par exemple, ceux décrits avec les termes énergétiques d'un système fermé comme le mouvement d'un pendule simple ou bien des phénomènes qui sont décrits en priorité par d'autres cadres énergétiques (par exemple, le principe des travaux virtuels) comme le fonctionnement du plan incliné en tant que machine simple (Koliopoulos, 1997). Au contraire, certaines catégories de phénomènes considérées comme privilégiées pour l'application du modèle conceptuel proposé, sont des phénomènes décrits en priorité par des modèles d'un système thermodynamique ouvert (où les élèves reconnaissent une source d'énergie et un récepteur d'énergie évidents, comme dans le fonctionnement d'un circuit électrique simple) ou bien des situations transphénoménologiques (comme le fonctionnement d'un modèle de machine thermique, par exemple l'allumage d'une lampe à l'aide d'une dynamo, la remontée d'un corps (Lemeignan \& Weil-Barais, 1990 ; Tiberghien \& Megalakaki, 1995) ou encore des phénomènes mécaniques comme la déformation d'un ressort par un objet quelconque (Koliopoulos, 1997).

Pour récapituler, nous présentons dans le tableau qui suit les principaux objectifs didactiques et les principales caractéristiques du contenu conceptuel qui en découlent, et cela dans les trois sortes de curriculums. 


\begin{tabular}{|c|c|c|c|}
\hline & Curriculum traditionnel & Curriculum innovatif & Curriculum constructiviste \\
\hline $\begin{array}{l}\text { Intentions et objectifs } \\
\text { didactiques } \\
\text { principaux }\end{array}$ & \begin{tabular}{|l|} 
- Il n'existe pas de rapport entre l'actuelle \\
analyse épistémologique du contenu de la \\
science, les exigences sociales et les \\
facteurs psychologiques desquels \\
semblent dépendre l'apprentissage des \\
concepts physiques. \\
- Ils sont en rapport principalement pour \\
ne pas dire exclusivement avec le contenu \\
de la science.
\end{tabular} & $\begin{array}{l}\text { - Ils découlent de l'analyse épistémologique du } \\
\text { contenu du concept d'énergie } \\
\text { - Ils se réfèrent non seulement à l'importance } \\
\text { principale du concept en sciences physiques mais } \\
\text { aussi à l'intérêt provenant des problèmes sociaux. }\end{array}$ & $\begin{array}{l}\text { - Ils proviennent de l'étude des conceptions des élèves } \\
\text { concernant l'énergie, alors que souvent coexistent } \\
\text { l'analyse épistémologique systématique du contenu du } \\
\text { concept en sciences physiques et la référence aux } \\
\text { exigences sociales et aux pratiques. } \\
\text { - La formulation est en général réalisée de façon à ce } \\
\text { qu'apparaisse le processus d'élaboration conceptuelle } \\
\text { sans pourtant qu'il y ait un seul type de formulation. }\end{array}$ \\
\hline $\begin{array}{l}\text { Caractéristiques } \\
\text { principales de la } \\
\text { structure et du } \\
\text { contenu }\end{array}$ & $\begin{array}{l}\text { - Dispersion du concept d'énergie en } \\
\text { diverses unités thématiques } \\
\text { conceptuelles où lui est attribué un sens } \\
\text { systémique et empirique différent. } \\
\text { - L'énergie est en général étudiée en tant } \\
\text { que concept dérivé du travail dans le } \\
\text { cadre de la Mécanique Newtonienne, où a } \\
\text { lieu l'étude principale. } \\
\text { - Choix fortuit du champ d'application du } \\
\text { concept d'énergie. }\end{array}$ & $\begin{array}{l}\text { - L'énergie constitue une large unité conceptuelle } \\
\text { ou un principe organisateur de tout le curriculum. } \\
\text { - L'énergie est introduite en tant que concept } \\
\text { premier, où est accentué le caractère unificateur et } \\
\text { trans-phénoménologique qui est assuré à travers } \\
\text { les concepts de transfert, de transformation et de } \\
\text { conservation de l'énergie. } \\
\text { - Le concept d'énergie acquiert en général une } \\
\text { signification unique pour toutes les unités } \\
\text { thématiques, puisqu'il est désormais intégré dans } \\
\text { un système conceptuel unique. } \\
\text { - Le champ d'application du concept d'énergie } \\
\text { contribue à la compréhension du caractère } \\
\text { unificateur et trans-phénoménologique du concept } \\
\text { d'énergie ainsi qu'à la description et à } \\
\text { l'interprétation des situations et problèmes } \\
\text { physiques qui proviennent de l'environnement } \\
\text { social familier. }\end{array}$ & $\begin{array}{l}\text { - L'énergie exige une unité conceptuelle unique où une } \\
\text { élaboration progressive est tentée, soit à travers la } \\
\text { différenciation des concepts énergétiques, soit à travers } \\
\text { l'introduction d'un "modèle-germe». } \\
\text { - La séquence des unités conceptuelles, le modèle } \\
\text { conceptuel de négociation du concept d'énergie et son } \\
\text { champ d'application sont influencés surtout par les } \\
\text { résultats de la recherche relatifs aux conceptions des } \\
\text { élèves. }\end{array}$ \\
\hline
\end{tabular}

Tableau 1: Les principaux objectifs didactiques et les principales caractéristiques du contenu conceptuel dans les trois sortes de curriculums 


\section{DISCUSSION}

Le cadre constitué par la classification proposée semble pouvoir offrir certaines possibilités à la tentative de réduire les distances entre la recherche en Didactique des sciences physiques et les pratiques pédagogiques dans l'enseignement des sciences physiques. Une approche de cette classification à travers ce point de vue offre la possibilité de mettre en valeur certains éléments qui constituent des champs d'intérêt commun et qui permettent la création d'un cadre de communication, puisqu'ils favorisent la lecture du réel à travers l'élaboration et la transformation d' «un contexte bien particulier de dialogue, appelé système commun de significations" (Amigues et Caillot, 1990).

Par exemple, les axes principaux de la classification nous ont permis les choix pertinents pour la construction d'un curriculum sur l'énergie mécanique pour des élèves de 13 - 14 ans qui s'inscrit dans une perspective de rénovation des programmes (Koliopoulos \& Ravanis, 2000a). Cette proposition concerne l'intégration de l'aspect constructiviste dans l'organisation et le contenu du curriculum lui-même. Sur la base de principes argumentés, c'est à dire (a) la prise en compte des conceptions des élèves (point d'appui pour une évolution), (b) un travail sur les systèmes symboliques et (c) la proposition de «germes de modèle» (Tiberghien \& Megalakaki, 1995), nous proposons une organisation temporelle de l'introduction de différents concepts associés au concept d'énergie, en indiquant les situations physiques qui paraissent les plus appropriées. A partir de cette proposition curriculaire, des séquences de classe ont été mises en oeuvre qui ont donné lieu à une évaluation de la validité du curriculum. Cette évaluation du produit de l'apprentissage a été effectuée par un questionnaire avant et après enseignement avec les mêmes questions, destiné à une centaine d'élèves. L'étude des justifications des élèves au pré-test montre que, à propos des phénomènes mécaniques en question, aucun élève ne présente de conception compatible avec des concepts énergétiques (des conceptions pré-énergétiques par exemple) ni n'utilise le terme d'énergie (ne serait-ce qu'avec une des formes alternatives présentées dans la bibliographie (Driver et al., 1994). La non apparition de telles conceptions est due, au fait que, comme nous l'avons déjà noté, les élèves n'expriment pas spontanément de telles conceptions quand ils décrivent et/ou expliquent des phénomènes mécaniques. Il semblerait donc que l'intervention didactique devienne une condition sine qua non à la formation par les élèves de conceptions pré-énergétiques évoluant ensuite vers des conceptions énergétiques.

La nécessité de l'approche entre l'enseignement scientifique et la Didactique des Sciences Physiques nous amène à étudier les conceptions des enseignants sur différents aspects de l'enseignement des Sciences Physiques pour les rendre conscientes et utilisables simultanément par l'enseignant et le chercheur. Ainsi, dans une recherche relative, la classification évoquée est appliquée à une étude des conceptions des enseignants scientifiques en formation qui planifient une série d'activités expérimentales concernant le concept d'énergie (Koliopoulos \& Ravanis, 1998). Les chercheurs ont constaté que la plupart des enseignants ont semblé avoir compris l'utilité d'un tel outil dans leurs pratiques pédagogiques. La discussion a abouti à la formulation de quatre constats importants de la part des enseignants : (a) les enseignants ont pris conscience des caractéristiques, des composantes et des limites du cadre dans lequel ils travaillent, (b) ils se sont rendus compte du pouvoir séparateur de l'outil de classification qui conduit à l'élargissement de leurs choix, (c) la classification des curriculums leur a donné la possibilité de formaliser et expliquer leurs pratiques pédagogiques, et par conséquent de pouvoir justifier et soutenir avec des arguments ces pratiques et, dans certain cas, (d) la classification peut faciliter la planification et la réorganisation de leur enseignement.

En outre, dans un autre travail, nous avons étudié la pertinence des trois types de conceptions curriculaires pour contribuer à la propagation d'une culture «énergétique» vers les élèves dans le cadre de l'éducation formelle. Plus précisément, nous avons voulu voir si et comment chaque approche pourrait conduire, à la fois, à l'appropriation des modèles explicatifs pouvant fonctionner au niveau des situations scolaires ainsi qu'au niveau de la vie de tous les jours et à la formation d'une conscience énergétique du citoyen ayant un rapport avec des problèmes contemporains comme, par exemple, la maîtrise de l'énergie à la maison et la gestion des ressources énergétiques (Koliopoulos \& Ravanis, 2000b). Notre analyse a montré que la conception innovative, au niveau de la planification apparaît comme une approche compatible à la formation 
d'une culture énergétique chez les élèves. En réalité, ce caractère unificateur du concept de l'énergie dans les curriculums de ce type pourrait permettre aux enfants la reconnaissance et la manipulation de problèmes énergétiques dans les différents domaines de la matière enseignée. C'est à dire qu'il valoriserait des possibilités que n'offre pas la conception traditionnelle qui amène des confusions diverses au niveau scientifique et épistémologique. Malgré ces présuppositions de départ positives, l'approche innovative reste faible au niveau de la construction cognitive des connaissances énergétiques opérationnelles, étant donné que les hypothèses d'apprentissage de cette approche ne sont pas suffisamment élaborées. D'autre part, l'approche constructiviste permet aux élèves la construction des liens entre champs phénoménologiques divers, en facilitant simultanément le passage des aspects qualitatifs aux aspects quantitatifs du concept (Koliopoulos \& Ravanis, 2000a). Ces aspects de l'approche constructiviste offrent aux curriculums qu'ils animent des possibilités qui apparaissent comme des conditions nécessaires mais pas suffisantes pour la formation éventuelle d'une culture énergétique chez les élèves parce que la dimension de l'appropriation du concept scientifique de l'énergie est un seul paramètre de la culture énergétique parmi d'autres d'ordre social, environnemental, politique et économique.

Évidemment, la classification en question a une portée limitée, compte tenu du fait qu'elle a été constituée uniquement en fonction de l'étude des programmes d'enseignement de l'énergie. De ce point de vue, la tentative de recherche des trois formes de curriculum (traditionnel, innovatif et constructiviste) présenterait probablement un intérêt particulier dans d'autres champs de sciences physiques également. Ainsi, dans la perspective d'une généralisation éventuelle du modèle, nous étudions son efficacité et sa pertinence comme outil de travail pour analyser des curriculums concernant le domaine de la Mécanique et plus spécialement le domaine du pendule (Koliopoulos \& Constantinou, 2005). Et si cette classification apparaissait fructueuse dans d'autres parties de curriculums, nous aurions alors des indications précises quant à sa valeur heuristique.

\section{BIBLIOGRAPHIE}

AGABRA J. et al. (1979). Sciences Physiques (Collection Libres Parcours). Paris. Hachette.

AGABRA J. (1986). Échanges thermiques. ASTER, 2, 1-41.

AMIGUES R. \& Caillot M. (1990). Les représentations graphiques dans l'enseignement et l'apprentissage de l'électricité. European Journal of Psychology of Education, V, 4, 477-488.

ARONS A.B. (1990). A guide to introductory Physics learning. New York. J. Wiley \& Sons.

ASTOLFI J.P. \& DEVELAY M. (1989). La didactique des sciences. Paris. Presses Universitaires de France.

BALTAS A. (1990). Once again on the meaning of physical concepts. In Nikolakopoulos P. (Ed.) Greek studies in the Philosophy and History of Science. Dordrecht. Kluwer Academic Publishers, 293-313.

GABORIEAU O. (1994). Physique-Chimie 3ème. Paris, Nathan.

DEVELAY M. (1992). De l'apprentissage à l'enseignement. Pour une épistémologie scolaire. Paris. ESF Editeur.

DRIVER R., SQUIRES A., RUSHWORTH P. \& WOOD-ROBINSON V. (1994). Making sense of secondary science. Research into children's ideas. London. Routledge.

DURANDEAU J.P. (1994). Sciences Physiques 3ème. Paris, Hachette.

FALK G. \& HERMANN F. (1981). Neue Physik, Das energiebuch. Hannover. Schroedel.

HABER-SCHAIM U. (1983). Energy. N. Jersey. Prentice-Hall, Inc., Englewood Cliffs.

HALBWACHS F. (1973). L'Histoire de l'explication en Physique. In J. Piaget et al., L'Explication dans les Sciences. Paris. Flammarion, 72-102.

JOHNSON K. \& A. (1991). Physics for you. London, Stanley Thornes Publishers Ltd.

KARIOTOGLOU P., PSILLOS D. \& VALASSIADES O. (1990). Understanding pressure : didactical transposition and pupils' conceptions. Physics Education, 25, 92-96.

KOLIOPOULOS D. (1997). Approches épistémologiques et didactiques du processus de construction de curriculum : Le cas de la transposition didactique et de l'apprentissage du concept d'énergie. Thèse. Patras, Université de Patras (http://www.summerschool.dk/esera/diss/dissgrece.html). 
KOLIOPOULOS D. \& RAVANIS K. (1998). L'enseignement de l'énergie au collège vu par les enseignants. Grille d'analyse de leurs conceptions. ASTER, 26, 165-182.

KOLIOPOULOS D. \& RAVANIS K. (1999). La classification du contenu conceptuel des curriculums concernant l'énergie: vers une modélisation des points de vue sur l'enseignement de l'énergie. In Actes des premières rencontres scientifiques de l'ARDIST. Paris. ARDIST, 156-160.

KOLIOPOULOS D. \& RAVANIS K. (2000a). Elaboration et évaluation du contenu conceptuel d'un programme constructiviste concernant l'approche énergétique des phénomènes mécaniques. Didaskalia. 16, 33-56.

KOLIOPOULOS D. \& RAVANIS K. (2000b). Réflexions méthodologiques sur la formation d'une culture concernant le concept d'énergie à travers l'éducation formelle., Revue de Recherches en Education : Spirale, 26, 73-86.

KOLIOPOULOS D. \& CONSTANTINOU C. (2005). The simple pendulum in school science textbooks of Greece and Cyprus. Science \& Education, 14, 1, 59-73.

LEHRMAN R.L. (1973). Energy is not the ability to do work. The Physics Teacher, 11, 1, 15-18.

LEMEIGNAN G. \& WEIL-BARAIS A. (1993). Construire des concepts en Physique. Paris. Hachette Education.

LOUDEN W. \& WALLACE J. (1994). Knowing and teaching science: the constructivist paradox. International Journal of Science Education, 16, 6, 649-657.

MARTINAND J.L. (1994). La Didactique des Sciences et de la Technologie et la formation des enseignants. ASTER, 19, 6175.

SHADMI Y et al. (1978). Investigating the concept of energy in the 9th grade. The Hebrew University of Jerusalem and the Curriculum Development Center (Education Ministry of Israel).

SHIPSTONE D.M. \& Gunstone R.F. (1985). Teaching children to discriminate between current and energy. In R. Duit et al. (Eds.), Aspects of understanding electricity. Kiel. IPN, University of Kiel, 287-297.

SOLOMON J. (1992). Getting to know about energy - in school and society. London. The Falmer press.

TIBERGHIEN A. (1989). Learning and teaching at middle school level of concepts and phenomena in physics: the case of temperature. In H. Mandl et al. (Eds.), Learning and Instruction: European research in an International context. Oxford. Pergammon Press, 631-648.

TIBERGHIEN A. \& MEGALAKAKI O. (1995). Characterisation of a modelling activity for a first qualitative approach to the concept of energy. European Journal of Psychology of Education, X, 4, 369-383.

ZENAKOS A. et al. (1994). Physique. Athènes (en grec).

DATA DE RECEBIMENTO: 12/05/05

DATA DE APROVAÇÃO:25/09/05 